Article

\title{
Approximation of the Fixed Point for Unified Three-Step Iterative Algorithm with Convergence Analysis in Busemann Spaces
}

\author{
Hassan Almusawa ${ }^{1}\left(\mathbb{D}\right.$, Hasanen A. Hammad ${ }^{2, *} \mathbb{D}$ and Nisha Sharma ${ }^{3} \mathbb{D}$ \\ 1 Department of Mathematics, College of Sciences, Jazan Univesity, Jazan 45142, Saudi Arabia; \\ almusawah@mymail.vcu.edu \\ 2 Department of Mathematics, Faculty of Science, Sohag University, Sohag 82524, Egypt \\ 3 Department of Mathematics, Pt. J.L.N. Govt. College, Faridabad 121002, India; nnishaa.bhardwaj@gmail.com \\ * Correspondence: hassanein_hamad@science.sohag.edu.eg
}

\section{check for}

updates

Citation: Almusawa, H.; Hammad, H.A.; Sharma, N. Approximation of the Fixed Point for Unified Three-Step Iterative Algorithm with Convergence Analysis in Busemann Spaces. Axioms 2021, 10, 26. https:// doi.org/10.3390/axioms10010026

Received: 12 January 2021

Accepted: 23 February 2021

Published: 27 February 2021

Publisher's Note: MDPI stays neutral with regard to jurisdictional claims in published maps and institutional affiliations.

Copyright: (c) 2021 by the authors Licensee MDPI, Basel, Switzerland. This article is an open access article distributed under the terms and conditions of the Creative Commons Attribution (CC BY) license (https:// creativecommons.org/licenses/by/ $4.0 /)$.

\begin{abstract}
In this manuscript, a new three-step iterative scheme to approximate fixed points in the setting of Busemann spaces is introduced. The proposed algorithms unify and extend most of the existing iterative schemes. Thereafter, by making consequent use of this method, strong and $\Delta$ convergence results of mappings that satisfy the condition $\left(\mathcal{E}_{\mu}\right)$ in the framework of uniformly convex Busemann space are obtained. Our results generalize several existing results in the same direction.
\end{abstract}

Keywords: the condition $\left(\mathcal{E}_{\mu}\right)$; standard three-step iteration algorithm; fixed point; uniformly convex Busemann space

MSC: AMS Subject Classification: 47H09; 47H10; 54H25; 54E40

\section{Introduction}

Throughout this paper, $\mathcal{R}, \mathcal{R}^{+}$, and $f_{\wp}$ denote the set of all real numbers, positive real numbers, and fixed points of the mapping $\wp$, respectively.

The fixed point theory is considered one of the most powerful analytical techniques in mathematics, especially in nonlinear analysis, where it plays a prominent role in algorithm technology. The purpose of investing in algorithms is to obtain the best algorithms with a faster convergence rate, because the lower the convergence rate, the faster the speed of obtaining the solution. This is probably the drawback of using the iterative methods.

It should be noted that the Mann iteration converges faster than the Ishikawa iteration for the class of Zamfirescu operators [1], and hence the convergence behavior of proclaimed and empirically proven faster iterative schemes need not always be faster. There was extensive literature on proclaimed new and faster iteration schemes in ancient times. Some of the iteration schemes are undoubtedly better versions of previously existed iteration schemes, whereas a few are only the special cases. There are more than twenty iteration schemes in the present literature. Our analysis's focal objective is to unify the existing results in the framework of Busemann spaces (see [2] for the precise definitions and properties of Busemann spaces). This analysis has a special significance in terms of unification, and numerous researchers have intensively investigated various aspects of it.

Apart from Picard, Mann, and Ishikawa, many iterative schemes with better convergence rates are obtained; see, for example, [3-11]. In many cases, these algorithms cannot obtain strong convergence; therefore, it was necessary to investigate new effective algorithms. Recently, several authors were able to apply the strong convergence of algorithms, see [12-16].

Recall that a metric space $(\mathcal{B}, \partial)$ is called a geodesic path (or simply a geodesic [17]) in $\mathcal{B}$ if there is a path $\gamma:[a, b] \rightarrow \mathcal{B}$, such that $\gamma$ is an isometry for $[a, b] \subset[0, \infty)$. A geodesic ray 
is an isometry $\gamma: \mathcal{R}^{+} \rightarrow \mathcal{B}$, and a geodesic line is an isometry $\gamma: \mathcal{R} \rightarrow \mathcal{B}$. For more details about geodesic path in metric fixed point theory, see [18-24].

Definition 1 ([17]). Let $(\mathcal{B}, \partial)$ be a metric space and $], \ell \in \mathcal{B}$. A geodesic path joining $\mathrm{f}$ to $\ell$ is a mapping $\gamma:[\alpha, \beta] \subseteq \mathcal{R}^{+} \rightarrow \mathcal{B}$ such that $\gamma(\alpha)=\jmath, \gamma(\beta)=\ell$ and

$$
\partial\left(\gamma(t), \gamma\left(t^{\prime}\right)\right)=\left|t-t^{\prime}\right|
$$

for all $t, t^{\prime} \in[\alpha, \beta]$. Particularly, $\gamma$ is an isometry and $\partial(\jmath, \ell)=\beta-\alpha$.

A geodesic segment joining $\mathrm{g}$ and $\ell$ in $\mathcal{B}$ is the image of a geodesic path in $\mathcal{B}$. The space $\mathcal{B}$ is said to be a geodesic space, if every two points of, are joined by a geodesic.

Definition 2 ([17]). A metric space $\mathcal{B}$ is said to be a geodesic space if given two arbitrary points of $\mathcal{B}$ there exists a geodesic path that joins them.

Definition 3 ([17]). The geodesic metric space $(\mathcal{B}, \partial)$ is said to be Busemann space, if for any two affinely reparametrized geodesices $\gamma:[\alpha, \beta] \rightarrow \mathcal{B}$ and $\gamma^{\prime}:\left[\alpha^{\prime}, \beta^{\prime}\right] \rightarrow \mathcal{B}$, the map $\mathcal{D}_{\gamma \cdot \gamma^{\prime}}$ : $[\alpha, \beta] \times\left[\alpha^{\prime}, \beta^{\prime}\right] \rightarrow \mathcal{R}$ defined by

$$
\mathcal{D}_{\gamma \cdot \gamma^{\prime}}\left(t, t^{\prime}\right)=\partial\left(\gamma(t), \gamma^{\prime}\left(t^{\prime}\right)\right)
$$

is a convex; that is, the metric of Busemann space is convex. In a Busemann space the geodesic joining any two points is unique.

Proposition 1 ([25]). In such spaces, the hypotheses below hold:

(1) $\partial(\varepsilon,(1-\alpha) \jmath \oplus \alpha \ell) \leq(1-\alpha) \partial(\varepsilon, \jmath)+\alpha \partial(\varepsilon, \ell)$,

(2) $\left.\partial((1-\alpha) \jmath \oplus \alpha \ell),\left(1-\alpha^{\prime}\right) \jmath \oplus \alpha^{\prime} \ell\right)=\left|\alpha-\alpha^{\prime}\right| \partial(\jmath, \ell)$,

(3) $(1-\alpha) \jmath \oplus \alpha \ell=\alpha \ell \oplus(1-\alpha)$,

(4) $\partial((1-\alpha) \jmath \oplus \alpha \varepsilon,((1-\alpha) \ell \oplus \alpha \omega)) \leq(1-\alpha) \partial(\jmath, \ell)+\alpha \partial(\varepsilon, \omega)$,

where $), \ell, \varepsilon, \omega \in \mathcal{B}$ and $\alpha, \alpha^{\prime} \in[0,1]$.

Busemann spaces are also hyperbolic spaces, which were introduced by Kohlenbach [26]. Further, $\mathcal{B}$ is said to be uniquely geodesic [17] if there is exactly one geodesic joining $f$ and $\ell$ for each,$\ell \in \mathcal{B}$.

Definition 4 ([17]). Suppose that $\mathcal{B}$ is a uniquely geodesic space and $\gamma([\alpha, \beta])$ is a geodesic segment joining $\mathrm{f}$ and $\ell$ and $\alpha \in[0,1]$. Then,

$$
\varepsilon=\gamma((1-\alpha) j+\alpha \ell)
$$

will be a unique point in $\gamma([\alpha, \beta])$ satisfying

$$
\partial(\varepsilon, \jmath)=\alpha \partial(\jmath, \ell)
$$

and

$$
\partial(\varepsilon, \ell)=(1-\alpha) \partial(\jmath, \ell) .
$$

In the sequel, the notation $[j, \ell]$ is used for geodesic segment $\gamma([\alpha, \beta])$ and $\varepsilon$ is denoted by $(1-\alpha) \jmath \oplus \alpha \ell$. A subset $\kappa \subseteq \mathcal{B}$ is said to be geodesically convex if $\kappa$ includes every geodesic segment joining any two of its points. Let $\mathcal{B}$ be a geodesic metric space and $\wp: \mathcal{B} \rightarrow \mathcal{R}$. We say that $\wp$ is convex if for every geodesic path $\gamma:[\alpha, \beta] \rightarrow \mathcal{B}$, the map $\wp \circ \gamma:[\alpha, \beta] \rightarrow$ is a convex. It is known that if $\wp: \mathcal{B} \rightarrow \mathcal{R}$ is a convex function and $\wp^{\prime}: \mathcal{B} \rightarrow \mathcal{R}$ is an increasing convex function, then $\wp^{\prime} \circ \wp: \mathcal{B} \rightarrow \mathcal{R}$ is convex.

We now introduce our algorithm. 
Let $\mathcal{B}$ be a complete Busemann space, $\mathcal{B}_{s}$ be a nonempty convex subset of $\mathcal{B}$ and $\wp: \mathcal{B}_{s} \rightarrow \mathcal{B}_{s}$ be a mapping. For any $v_{0} \in \mathcal{B}_{s}$,

$$
\left\{\begin{array}{l}
\left.\ell_{\eta}=\tau_{\eta}^{0} v_{\eta} \oplus \kappa_{\eta}^{0} \wp v_{\eta} \oplus \iota_{\eta}^{0}\right]_{\eta} \oplus \omega_{\eta}^{0} \wp J_{\eta}, \\
\jmath_{\eta}=\tau_{\eta}^{1} v_{\eta} \oplus \kappa_{\eta}^{1} \wp v_{\eta} \oplus \iota_{\eta}^{1} \ell_{\eta} \oplus \omega_{\eta}^{1} \wp \ell_{\eta}, \\
\left.v_{n+1}=\tau_{\eta}^{2} v_{\eta} \oplus \kappa_{\eta}^{2} \wp v_{\eta} \oplus i_{\eta}^{2}\right]_{\eta} \oplus \omega_{\eta}^{2} \wp J_{\eta} \oplus \varepsilon_{\eta} \ell_{\eta} \oplus \sigma_{\eta} \wp \ell_{\eta},
\end{array}\right.
$$

where $\left\{\varepsilon_{\eta}\right\},\left\{\sigma_{\eta}\right\},\left\{\tau_{\eta}^{i}\right\},\left\{\kappa_{\eta}^{i}\right\},\left\{\iota_{\eta}^{i}\right\}$ and $\left\{\omega_{\eta}^{i}\right\}$ for $i=0,1,2$ are sequences in $[0,1]$. Moreover, $\tau_{\eta}^{0}+\kappa_{\eta}^{0}+\iota_{\eta}^{0}+\omega_{\eta}^{0}=1, \tau_{\eta}^{1}+\kappa_{\eta}^{1}+\iota_{\eta}^{1}+\omega_{\eta}^{1}=1$ and $\tau_{\eta}^{2}+\kappa_{\eta}^{2}+\iota_{\eta}^{2}+\varepsilon_{\eta}+\sigma_{\eta}=1$.

Remark 1. For distinct values of $\varepsilon_{\eta},\left\{\sigma_{\eta}\right\}, \tau_{\eta}^{i}, \kappa_{\eta}^{i}, \iota_{\eta}^{i}$ and $\omega_{\eta}^{i}$ for $i=0,1,2$, we have well-known distinct iteration schemes as follows:

$\left(\mathcal{R}_{1}\right) \iota_{\eta}^{0}=\omega_{\eta}^{0}=\iota_{\eta}^{1}=\kappa_{\eta}^{1}=\iota_{\eta}^{2}=\kappa_{\eta}^{2}=\varepsilon_{\eta}=\sigma_{\eta}=0, \tau_{\eta}^{0}=\left(1-\kappa_{\eta}^{0}\right), \tau_{\eta}^{1}=\left(1-\omega_{\eta}^{1}\right)$, $\tau_{\eta}^{1}=\left(1-\omega_{\eta}^{2}\right)$ in the standard three-step iteration scheme, we obtain the Noor iterative scheme [27].

$\left(\mathcal{R}_{2}\right) \iota_{\eta}^{0}=\omega_{\eta}^{0}=\iota_{\eta}^{1}=\tau_{\eta}^{1}=\kappa_{\eta}^{1}=\iota_{\eta}^{2}=\omega_{\eta}^{2}=\varepsilon_{\eta}=\sigma_{\eta}=0, \tau_{\eta}^{0}=\left(1-\kappa_{\eta}^{0}\right), \iota_{\eta}^{1}=\left(1-\omega_{\eta}^{1}\right)$ and $\iota_{\eta}^{2}=\left(1-\omega_{\eta}^{2}\right)$ in the standard three-step iteration scheme, we obtain the SP iterative scheme [28].

$\left(\mathcal{R}_{3}\right) \iota_{\eta}^{0}=\omega_{\eta}^{0}=\iota_{\eta}^{1}=\tau_{\eta}^{1}=\kappa_{\eta}^{1}=\tau_{\eta}^{2}=\iota_{\eta}^{2}=\omega_{\eta}^{2}=\varepsilon_{\eta}=\sigma_{\eta}=0, \iota_{\eta}^{0}=\left(1-\kappa_{\eta}^{0}\right), \kappa_{\eta}^{1}=\left(1-\omega_{\eta}^{1}\right)$ and $\kappa_{\eta}^{2}=1$ in the standard three-step iteration scheme, we obtain the Picard-S iterative scheme [29].

$\left(\mathcal{R}_{4}\right) \tau_{\eta}^{0}=\kappa_{\eta}^{0}=\iota_{\eta}^{0}=\omega_{\eta}^{0}=\iota_{\eta}^{1}=\omega_{\eta}^{1}=\tau_{\eta}^{2}=\kappa_{\eta}^{2}=\varepsilon_{\eta}=\sigma_{\eta}=0$ and $\tau_{\eta}^{0}=\left(1-\kappa_{\eta}^{0}\right)$, $\kappa_{\eta}^{1}=\left(1-\omega_{\eta}^{1}\right)$ and $\iota_{\eta}^{2}=\left(1-\omega_{\eta}^{2}\right)$ in the standard three-step iteration scheme, we obtain the CR iterative scheme [30].

$\left(\mathcal{R}_{5}\right) \tau_{\eta}^{0}=\kappa_{\eta}^{0}=\iota_{\eta}^{1}=\tau_{\eta}^{1}=\tau_{\eta}^{2}=\iota_{\eta}^{2}=\varepsilon_{\eta}=\sigma_{\eta}=0, \tau_{\eta}^{1}=\left(1-\kappa_{\eta}^{1}\right), \kappa_{\eta}^{1}=\left(1-\omega_{\eta}^{1}\right)$ in the standard three-step iteration scheme, we obtain the Abbas and Nazir iterative scheme [31].

$\left(\mathcal{R}_{6}\right) \iota_{\eta}^{0}=\omega_{\eta}^{0}=\tau_{\eta}^{1}=\kappa_{\eta}^{1}=\kappa_{\eta}^{2}=\iota_{\eta}^{2}=\omega_{\eta}^{2}=\varepsilon_{\eta}=0, \tau_{\eta}^{0}=\left(1-\kappa_{\eta}^{0}\right), \iota_{\eta}^{1}=\left(1-\omega_{\eta}^{1}\right)$ and $\sigma_{\eta}^{2}=\left(1-\omega_{\eta}^{2}\right)$ in the standard three-step iteration scheme, we obtain the P iterative scheme [32].

$\left(\mathcal{R}_{7}\right) \iota_{\eta}^{0}=\omega_{\eta}^{0}=\tau_{\eta}^{1}=\iota_{\eta}^{1}=\kappa_{\eta}^{2}=\iota_{\eta}^{2}=\tau_{\eta}^{2}=\varepsilon_{\eta}=0, \tau_{\eta}^{0}=\left(1-\kappa_{\eta}^{0}\right), \kappa_{\eta}^{1}=\left(1-\omega_{\eta}^{1}\right)$ and $\sigma_{\eta}^{2}=\left(1-\omega_{\eta}^{2}\right)$ in the standard three-step iteration scheme, we obtain the $D$ iterative scheme [33].

$\left(\mathcal{R}_{8}\right) \iota_{\eta}^{0}=\omega_{\eta}^{0}=\tau_{\eta}^{0}=\kappa_{\eta}^{0}=\iota_{\eta}^{1}=\omega_{\eta}^{1}=\tau_{\eta}^{1}=\kappa_{\eta}^{1}=\omega_{\eta}^{2}=\iota_{\eta}^{2}=\varepsilon_{\eta}=0, \tau_{\eta}^{2}=\left(1-\kappa_{\eta}^{2}\right)$ in the standard three-step iteration scheme, we obtain the Mann iterative scheme [34].

$\left(\mathcal{R}_{9}\right) \iota_{\eta}^{0}=\omega_{\eta}^{0}=\tau_{\eta}^{0}=\kappa_{\eta}^{0}=\iota_{\eta}^{1}=\omega_{\eta}^{1}=\kappa_{\eta}^{2}=\iota_{\eta}^{2}=\varepsilon_{\eta}=\sigma_{\eta}=0, \tau_{\eta}^{1}=\left(1-\kappa_{\eta}^{1}\right)$ and $\tau_{\eta}^{2}=\left(1-\omega_{\eta}^{2}\right)$ in the standard three-step iteration scheme, we obtain the Ishikawa iterative scheme [35].

\section{Preliminaries}

In this section, we present some relevant and essential definitions, lemmas, and theorems needed in the sequel.

Definition 5 ([36]). The Busemann space $\mathcal{B}$ is called uniformly convex if for any $\zeta>0$ and $\epsilon \in(0,2]$, there exists a map $\delta$ such that for every three points $\alpha, \jmath, \ell \in \mathcal{B}, \partial(\jmath, \alpha) \leq \zeta, \partial(\ell, \alpha) \leq \zeta$ and $\partial(j, \ell) \geq \epsilon \zeta$ implies that

$$
\partial(m, \alpha) \leq(1-\delta) \zeta
$$

where $m$ denotes the midpoint of any geodesic segment $[$ j, $\ell]$ (i.e., $m=\frac{1}{2} \rho \oplus \frac{1}{2} \ell$ ) and $\inf \{\delta: \zeta>0\}$. A mapping $\wp:(0, \infty) \times(0,2] \rightarrow(0,1]$ is called a modulus of uniform convexity, for $\wp(\eta, \epsilon):=\delta$ and for a given $\eta>0, \epsilon \in(0,2]$.

Henceforth, the uniform convexity modulus with a decreasing modulus concerning $\eta$ (for a fixed $\epsilon$ ) is termed as the uniform convexity monotone modulus. The subsequent 
lemmas and geometric properties, which are instrumental throughout the discussion to learn about essential terms of Busemann spaces, are necessary to achieve our significant findings and are as follows:

Lemma 1. If $\wp$ is a mapping satisfying condition $(\mathcal{E})$ and has a fixed point then it is a quasinonexpansive mapping.

Let $\mathcal{B}_{S}$ be a nonempty closed convex subset of a Busemann space $\mathcal{B}$, and let $\left\{\jmath_{\eta}\right\}$ be a bounded sequence in $\mathcal{B}$. For $\jmath \in \mathcal{B}$, we set

$$
\zeta\left(j,\left\{j_{\eta}\right\}\right)=\limsup _{\eta \rightarrow \infty}\|\|_{\eta}-\jmath \| .
$$

The asymptotic radius of $\zeta\left(\left\{J_{\eta}\right\}\right.$ is given by

$$
\zeta\left(\mathcal{B}_{s},\left\{J_{\eta}\right\}\right)=\inf \left\{\zeta\left(\jmath,\left\{J_{\eta}\right\}\right): \jmath \in \mathcal{B}_{s}\right\}
$$

and the asymptotic center $\mathcal{A}\left(\left\{\jmath_{\eta}\right\}\right)$ of $\left\{\jmath_{\eta}\right\}$ relative to $\mathcal{B}_{s}$ is the set

$$
\mathcal{A}\left(\mathcal{B}_{s},\left\{\jmath_{\eta}\right\}\right)=\left\{\jmath \in \mathcal{B}_{s}: \zeta\left(\jmath, \jmath_{\eta}\right)=\zeta\left(\mathcal{B}_{s},\left\{\jmath_{\eta}\right\}\right)\right\} .
$$

It is known that, in a Busemann space, $\mathcal{A}\left(\left\{j_{\eta}\right\}\right)$ consists of exactly one point [37].

Recall that a bounded sequence $\left\{j_{\eta}\right\} \in \mathcal{B}$ is said to be regular [38], if $\zeta\left(\left\{j_{\eta}\right\}\right)=$ $\zeta\left(\left\{\eta_{\eta_{k}}\right\}\right)$ for every subsequence $\left\{\eta_{\eta_{k}}\right\}$ of $\left\{\eta_{\eta}\right\}$.

Lemma 2 ([4]). Let $\mathcal{B}$ be a Busemann space and $] \in \mathcal{B},\left\{t_{n}\right\}$ a sequence in $[b, c]$, for some $b, c \in(0,1)$. If $\left\{j_{\eta}\right\}$ and $\left\{\ell_{\eta}\right\}$ are sequences in $\mathcal{B}$ satisfying

$$
\limsup _{\eta \rightarrow \infty} \partial\left(\jmath_{\eta}, \jmath\right) \leq r
$$

also,

$$
\limsup _{\eta \rightarrow \infty} \partial\left(\ell_{\eta}, \ell\right) \leq r
$$

and

$$
\limsup _{\eta \rightarrow \infty} \partial\left(t_{\eta} \jmath_{\eta} \oplus\left(1-t_{\eta}\right) \ell_{\eta}, \jmath\right)=r
$$

for some $r \geq 0$, then

$$
\lim _{\eta \rightarrow \infty} \partial\left(\jmath_{\eta}, \ell_{\eta}\right)=0
$$

Lemma 3 ([5]). If $\mathcal{B}_{s}$ is a closed convex subset of a uniformly convex Busemann space $\mathcal{B}$ and \{]$\left._{\eta}\right\}$ is a bounded sequence in $\mathcal{B}_{s}$, then the asymptotic center of $\left\{j_{\eta}\right\}$ belongs to $\mathcal{B}_{s}$.

Lemma 4 ([38]). Let $\mathcal{B}$ be a Busemann space, $\left\{\jmath_{\eta}\right\}$ be a bounded sequence in $\mathcal{B}$ and $\mathcal{B}_{s}$ be a subset of $\mathcal{B}$. Then $\left\{j_{\eta}\right\}$ has a subsequence, which is regular in $\mathcal{B}_{S}$.

Definition 6 ([38]). A sequence $\left\{J_{\eta}\right\}$ in Busemann space $\mathcal{B}$ is said to be $\Delta-$ convergent if there exists some $j \in \mathcal{B}$ such that $j$ is the unique asymptotic center of $j_{\eta_{k}}$ for every subsequence $\left\{\eta_{\eta_{k}}\right\}$ of $\left\{\eta_{\eta}\right\}$. In this case we write $\left.\Delta-\lim _{\eta \rightarrow \infty}\right]_{\eta}=j$ and it is called the $\Delta-\lim$ of $\left\{\eta_{\eta}\right\}$.

Lemma 5 ([21]). Every bounded sequence in a complete Busemann space always has a $\Delta-$ convergent subsequence.

Lemma 6 ([21]). Suppose that $\mathcal{B}_{s}$ is a closed convex subset of a Busemann space $\mathcal{B}$ and $\wp: \mathcal{B}_{s} \rightarrow \mathcal{B}$ satisfies the condition $(\mathcal{E})$. Then $\left\{\jmath_{\eta}\right\}, \Delta-$ converges to $j$ and $\partial\left(\wp_{\eta}, \jmath_{\eta}\right) \rightarrow 0$, implying that $\jmath \in \mathcal{B}_{s}$ 
and $\wp=\jmath$.

Definition 7. Assume that $\mathcal{B}_{s} \neq \varnothing$ is a subset of a Busemann space $\mathcal{B}$. For $], \ell \in \mathcal{B}_{s}$, a mapping $\wp: \mathcal{B}_{S} \rightarrow \mathcal{B}_{s}$ is called:

(i) Contraction if there is $\mu \in(0,1)$ so that $\partial(\wp), \wp \ell) \leq \mu \partial(j, \ell)$,

(ii) Nonexpansive if $\partial(\wp), \wp \ell) \leq \partial(\jmath, \ell)$,

(iii) Quasi-nonexpansive if $\partial(\wp), \varkappa) \leq \partial(\jmath, \varkappa), \varkappa \in F(\wp)$ and $F(\wp)$ denote the set $\{\varkappa \in \mathcal{B}$ : $\varkappa=\wp \varkappa\}$.

(iv) Satisfy Condition $(\mathcal{E})$ if

$$
\frac{1}{2} \partial(\jmath, \wp \jmath) \leq \partial(\jmath, \ell) \Rightarrow \partial(\wp \jmath, \wp \ell) \leq \partial(\jmath, \ell) .
$$

(v) Suzuki generalized nonexpansive if it verifies Condition $(\mathcal{E})$.

Garcia-Falset et al. [6] introduced the generalization for nonexpansive mappings known as condition $\left(\mathcal{E}_{\mu}\right)$.

Definition 8 ([6]). Let $\mu \geq 1$. A mapping $\wp: \mathcal{B}_{s} \rightarrow \mathcal{B}_{s}$ is said to satisfy condition $\left(\mathcal{E}_{\mu}\right)$ if for all ,$\ell \in \mathcal{B}_{s}$, we have

$$
\partial(\jmath, \wp \ell) \leq \mu \partial(\jmath, \wp \jmath)+\partial(\jmath, \ell) .
$$

We say that $\wp$ satisfies condition $(\mathcal{E})$, if $\wp$ satisfies condition $\left(\mathcal{E}_{\mu}\right)$ for some $\mu \geq 1$ [39].

Theorem 1. Let $\mathcal{C}_{S}$ be a nonempty bounded, closed and convex subset of a complete $C A T(0)$ space $\mathcal{C}$. If $\wp: \mathcal{C}_{S} \rightarrow \mathcal{C}_{S}$ is a generalized nonexpansive mapping, then $\wp$ has a fixed point in $\mathcal{C}_{s}$. Moreover, $f_{\wp}$ is closed and convex.

\section{Main Results}

We begin this section with the proof of the following lemmas:

Lemma 7. Let $\mathcal{B}_{s}$ be a nonempty closed convex subset of a complete Busemann space $\mathcal{B}$, and let $\wp: \mathcal{B}_{S} \rightarrow \mathcal{B}_{S}$ be a mapping satisfying condition $\left(\mathcal{E}_{\mu}\right)$. For an arbitrary chosen $v_{0} \in \mathcal{B}_{s}$, let the sequence $\left\{v_{\eta}\right\}$ be generated by a standard three-step iteration algorithm with the condition

$$
\left(\left(\tau_{\eta}^{1}+\kappa_{\eta}^{1}\right)+\left(\iota_{\eta}^{1}+\omega_{\eta}^{1}\right)\left(\tau_{\eta}^{0}+\kappa_{\eta}^{0}\right)\right)\left(1-\left(\iota_{\eta}^{1}+\omega_{\eta}^{1}\right)\left(\iota_{\eta}^{0}+\omega_{\eta}^{0}\right)\right)^{-1} \leq 1
$$

and

$$
\left(\tau_{\eta}^{2}+\kappa_{\eta}^{2}+\iota_{\eta}^{2}+\omega_{\eta}^{2}+\left(\varepsilon_{\eta}+\sigma_{\eta}\right)\left(\tau_{\eta}^{0}+\kappa_{\eta}^{0}+\iota_{\eta}^{0}+\omega_{\eta}^{0}\right)\right) \leq 1 .
$$

Then, $\lim _{n \rightarrow \infty} \partial\left(v_{\eta}, v_{*}\right)$ exists for all $v_{*} \in f_{\wp}$.

Proof. Let $v_{*} \in f_{\wp}$ and $z \in \mathcal{B}_{s}$. Since $\wp$ satisfies condition $\left(\mathcal{E}_{\mu}\right)$, and hence

$$
\partial\left(v_{*}, \wp \jmath\right) \leq \mu \partial\left(v_{*}, \wp v_{*}\right)+\partial\left(v_{*},\right) .
$$

From standard three-step iteration algorithm, we have

$$
\begin{aligned}
\partial\left(\ell_{\eta}, v_{*}\right) & =\partial\left(\tau_{\eta}^{0} v_{\eta} \oplus \kappa_{\eta}^{0} \wp v_{\eta} \oplus \iota_{\eta}^{0} \jmath_{\eta} \oplus \omega_{\eta}^{0} \wp \jmath_{\eta}, v_{*}\right) \\
& \leq \tau_{\eta}^{0} \partial\left(v_{\eta}, v_{*}\right)+\kappa_{\eta}^{0}\left(\mu \partial\left(v_{*}, \wp v_{*}\right)+\partial\left(v_{*}, v_{n}\right)\right)+\iota_{\eta}^{0} \partial\left(\jmath_{\eta}, v_{*}\right)+\omega_{\eta}^{0}\left(\mu \partial\left(v_{*}, \wp v_{*}\right)+\partial\left(v_{*}, \jmath_{n}\right)\right) \\
& \leq \tau_{\eta}^{0} \partial\left(v_{\eta}, v_{*}\right)+\kappa_{\eta}^{0} \partial\left(v_{\eta}, v_{*}\right)+\iota_{\eta}^{0} \partial\left(\jmath_{\eta}, v_{*}\right)+\omega_{\eta}^{0} \partial\left(\jmath_{\eta}, v_{*}\right) \\
& =\left(\tau_{\eta}^{0}+\kappa_{\eta}^{0}\right) \partial\left(v_{\eta}, v_{*}\right)+\left(\iota_{\eta}^{0}+\omega_{\eta}^{0}\right) \partial\left(\jmath_{\eta}, v_{*}\right) .
\end{aligned}
$$

Also, 


$$
\begin{aligned}
\partial\left(\jmath_{\eta}, v_{*}\right) & =\partial\left(\tau_{\eta}^{1} v_{\eta} \oplus \kappa_{\eta}^{1} \wp v_{\eta} \oplus \iota_{\eta}^{1} \ell_{\eta} \oplus \omega_{\eta}^{1} \wp \ell_{\eta}, v_{*}\right) \\
& \leq \tau_{\eta}^{1} \partial\left(v_{\eta}, v_{*}\right)+\kappa_{\eta}^{1} \partial\left(\wp v_{\eta}, v_{*}\right)+\iota_{\eta}^{1} \partial\left(\ell_{\eta}, v_{*}\right)+\omega_{\eta}^{1} \partial\left(\wp \ell_{\eta}, v_{*}\right) \\
& \leq \tau_{\eta}^{1} \partial\left(v_{\eta}, v_{*}\right)+\kappa_{\eta}^{1}\left(\mu \partial\left(v_{*}, \wp v_{*}\right)+\partial\left(v_{*}, v_{n}\right)\right)+\iota_{\eta}^{1} \partial\left(\ell_{\eta}, v_{*}\right)+\omega_{\eta}^{1}\left(\mu \partial\left(v_{*}, \wp v_{*}\right)+\partial\left(v_{*}, \ell_{n}\right)\right) \\
& =\left(\tau_{\eta}^{1}+\kappa_{\eta}^{1}\right) \partial\left(v_{\eta}, v_{*}\right)+\left(\iota_{\eta}^{1}+\omega_{\eta}^{1}\right) \partial\left(\ell_{\eta}, v_{*}\right) .
\end{aligned}
$$

Using the value of $\partial\left(\ell_{\eta}, v_{*}\right)$, we have

$$
\begin{aligned}
\partial\left(\jmath_{\eta}, v_{*}\right) & \leq\left(\tau_{\eta}^{1}+\kappa_{\eta}^{1}\right) \partial\left(v_{\eta}, v_{*}\right)+\left(\iota_{\eta}^{1}+\omega_{\eta}^{1}\right)\left(\left(\tau_{\eta}^{0}+\kappa_{\eta}^{0}\right) \partial\left(v_{\eta}, v_{*}\right)+\left(\iota_{\eta}^{0}+\omega_{\eta}^{0}\right) \partial\left(\jmath_{\eta}, v_{*}\right)\right) \\
& \leq\left(\left(\tau_{\eta}^{1}+\kappa_{\eta}^{1}\right)+\left(\iota_{\eta}^{1}+\omega_{\eta}^{1}\right)\left(\tau_{\eta}^{0}+\kappa_{\eta}^{0}\right)\right) \partial\left(v_{\eta}, v_{*}\right)+\left(\iota_{\eta}^{1}+\omega_{\eta}^{1}\right)\left(\iota_{\eta}^{0}+\omega_{\eta}^{0}\right) \partial\left(\jmath_{\eta}, v_{*}\right) \\
\partial\left(\jmath_{\eta}, v_{*}\right) & \leq\left(\frac{\left(\tau_{\eta}^{1}+\kappa_{\eta}^{1}\right)+\left(\iota_{\eta}^{1}+\omega_{\eta}^{1}\right)\left(\tau_{\eta}^{0}+\kappa_{\eta}^{0}\right)}{1-\left(\iota_{\eta}^{1}+\omega_{\eta}^{1}\right)\left(\iota_{\eta}^{0}+\omega_{\eta}^{0}\right)}\right) \partial\left(v_{\eta}, v_{*}\right) .
\end{aligned}
$$

Since $\left(\left(\tau_{\eta}^{1}+\kappa_{\eta}^{1}\right)+\left(\iota_{\eta}^{1}+\omega_{\eta}^{1}\right)\left(\tau_{\eta}^{0}+\kappa_{\eta}^{0}\right)\right)\left(1-\left(\iota_{\eta}^{1}+\omega_{\eta}^{1}\right)\left(\iota_{\eta}^{0}+\omega_{\eta}^{0}\right)\right)^{-1} \leq 1$, we have

$$
\partial\left(\jmath_{\eta}, v_{*}\right) \leq \partial\left(v_{\eta}, v_{*}\right) .
$$

Now,

$$
\begin{aligned}
\partial\left(v_{n+1}, v_{*}\right) & \leq \partial\left(\tau_{\eta}^{2} v_{\eta} \oplus \kappa_{\eta}^{2} \wp v_{\eta} \oplus \iota_{\eta}^{2} \jmath_{\eta} \oplus \omega_{\eta}^{2} \wp \jmath_{\eta} \oplus \varepsilon_{\eta} \ell_{\eta} \oplus \sigma_{\eta} \wp \ell_{\eta}, v_{*}\right) \\
& \leq \tau_{\eta}^{2} \partial\left(v_{\eta}, v_{*}\right)+\kappa_{\eta}^{2} \partial\left(\wp v_{\eta}, v_{*}\right)+\iota_{\eta}^{2} \partial\left(\jmath_{\eta}, v_{*}\right)+\omega_{\eta}^{2} \partial\left(\wp \jmath_{\eta}, v_{*}\right)+\varepsilon_{\eta} \partial\left(\ell_{\eta}, v_{*}\right)+\sigma_{\eta} \partial\left(\wp \ell_{\eta}, v_{*}\right) \\
& \leq \tau_{\eta}^{2} \partial\left(v_{\eta}, v_{*}\right)+\kappa_{\eta}^{2}\left(\mu \partial\left(\wp v_{*}, v_{*}\right)+\partial\left(v_{\eta}, v_{*}\right)\right)+\iota_{\eta}^{2} \partial\left(\jmath_{\eta}, v_{*}\right)+\omega_{\eta}^{2}\left(\mu \partial\left(\wp v_{*}, v_{*}\right)+\partial\left(\jmath_{\eta}, v_{\eta}\right)\right) \\
& +\varepsilon_{\eta} \partial\left(\ell_{\eta}, v_{*}\right)+\sigma_{\eta}\left(\mu \partial\left(\wp v_{*}, v_{*}\right)+\partial\left(\ell_{\eta}, v_{*}\right)\right) \\
& \leq\left(\tau_{\eta}^{2}+\kappa_{\eta}^{2}\right) \partial\left(v_{\eta}, v_{*}\right)+\left(\iota_{\eta}^{2}+\omega_{\eta}^{2}\right) \partial\left(j_{\eta}, v_{*}\right)+\left(\varepsilon_{\eta}+\sigma_{\eta}\right) \partial\left(\ell_{\eta}, v_{*}\right) .
\end{aligned}
$$

Since

$$
\partial\left(\jmath_{\eta}, v_{*}\right) \leq \partial\left(v_{\eta}, v_{*}\right),
$$

we have

$$
\partial\left(v_{n+1}, v_{*}\right) \leq\left(\tau_{\eta}^{2}+\kappa_{\eta}^{2}+\iota_{\eta}^{2}+\omega_{\eta}^{2}\right) \partial\left(v_{\eta}, v_{*}\right)+\left(\varepsilon_{\eta}+\sigma_{\eta}\right) \partial\left(\ell_{\eta}, v_{*}\right) .
$$

On substituting

$$
\partial\left(\ell_{\eta}, v_{*}\right)=\left(\tau_{\eta}^{0}+\kappa_{\eta}^{0}\right) \partial\left(v_{\eta}, v_{*}\right)+\left(\iota_{\eta}^{0}+\omega_{\eta}^{0}\right) \partial\left(\jmath_{\eta}, v_{*}\right),
$$

we have

$$
\begin{aligned}
\partial\left(v_{n+1}, v_{*}\right) & \leq\left(\tau_{\eta}^{2}+\kappa_{\eta}^{2}+\iota_{\eta}^{2}+\omega_{\eta}^{2}\right) \partial\left(v_{\eta}, v_{*}\right)+\left(\left(\varepsilon_{\eta}+\sigma_{\eta}\right) \times\left(\tau_{\eta}^{0}+\kappa_{\eta}^{0}\right) \partial\left(v_{\eta}, v_{*}\right)+\left(\iota_{\eta}^{0}+\omega_{\eta}^{0}\right) \partial\left(\jmath_{\eta}, v_{*}\right)\right) \\
& \leq\left(\tau_{\eta}^{2}+\kappa_{\eta}^{2}+\iota_{\eta}^{2}+\omega_{\eta}^{2}\right) \partial\left(v_{\eta}, v_{*}\right)+\left(\varepsilon_{\eta}+\sigma_{\eta}\right)\left(\tau_{\eta}^{0}+\kappa_{\eta}^{0}+\iota_{\eta}^{0}+\omega_{\eta}^{0}\right) \partial\left(v_{\eta}, v_{*}\right) \\
& =\left(\tau_{\eta}^{2}+\kappa_{\eta}^{2}+\iota_{\eta}^{2}+\omega_{\eta}^{2}+\left(\varepsilon_{\eta}+\sigma_{\eta}\right)\left(\tau_{\eta}^{0}+\kappa_{\eta}^{0}+\iota_{\eta}^{0}+\omega_{\eta}^{0}\right)\right) \partial\left(v_{\eta}, v_{*}\right) .
\end{aligned}
$$

Also, it is given that

$$
\left(\tau_{\eta}^{2}+\kappa_{\eta}^{2}+\iota_{\eta}^{2}+\omega_{\eta}^{2}+\left(\varepsilon_{\eta}+\sigma_{\eta}\right)\left(\tau_{\eta}^{0}+\kappa_{\eta}^{0}+\iota_{\eta}^{0}+\omega_{\eta}^{0}\right)\right) \leq 1,
$$

we have

$$
\partial\left(v_{n+1}, v_{*}\right) \leq \partial\left(v_{\eta}, v_{*}\right) .
$$

This implies that $\left\{\partial\left(v_{\eta}, v_{*}\right)\right\}$ is bounded and non-increasing for all $v_{*} \in f_{\wp}$. Hence, $\lim _{n \rightarrow \infty} \partial\left(v_{\eta}, v_{*}\right)$ exists, as required. 
Lemma 8. Let $\mathcal{B}_{S}$ be a nonempty closed convex subset of complete Busemann space $\mathcal{B}$, and $\wp: \mathcal{B}_{s} \rightarrow \mathcal{B}_{s}$ be a mapping satisfying condition $\left(\mathcal{E}_{\mu}\right)$. For an arbitrary chosen $v_{0} \in \mathcal{B}_{s}$, let the sequence $\left\{v_{\eta}\right\}$ be generated by a standard three-step iteration algorithm. Then, $f_{\wp}$ is nonempty if and only if $\left\{v_{\eta}\right\}$ is bounded and $\lim _{n \rightarrow \infty} \partial\left(\wp v_{\eta}, v_{\eta}\right)=0$ for a unique asymptotic center.

Proof. Since $f_{\wp} \neq \varnothing$, let $v_{*} \in f_{\wp}$ and $z \in \mathcal{B}_{s}$. Using Lemma 7 , there is an existence of $\lim _{n \rightarrow \infty} \partial\left(v_{\eta}, v_{*}\right)$, which confirms the boundedness of $\left\{v_{\eta}\right\}$. Assuming

$$
\lim _{n \rightarrow \infty} \partial\left(v_{\eta}, v_{*}\right)=r
$$

on combining this result with the values of $\partial\left(j_{\eta}, v_{*}\right)$ and $\partial\left(\ell_{\eta}, v_{*}\right)$ of Lemma 7

$$
\limsup _{n \rightarrow \infty} \partial\left(\ell_{\eta}, v_{*}\right) \leq \limsup _{n \rightarrow \infty} \partial\left(v_{\eta}, v_{*}\right)=r
$$

Also,

$$
\begin{aligned}
\limsup _{n \rightarrow \infty} \partial\left(\wp v_{\eta}, v_{*}\right) & =\limsup _{n \rightarrow \infty}\left(\mu \partial\left(\wp v_{*}, v_{*}\right)+\partial\left(v_{\eta}, v_{*}\right)\right) \\
& \leq \limsup _{n \rightarrow \infty} \partial\left(v_{\eta}, v_{*}\right) \\
& =r .
\end{aligned}
$$

On the other hand, by using the value of $\partial\left(\ell_{\eta}, v_{*}\right)$ of Lemma 7 , we have

$$
\begin{aligned}
\partial\left(v_{n+1}, v_{*}\right) & \leq \partial\left(\tau_{\eta}^{2} v_{\eta} \oplus \kappa_{\eta}^{2} \wp v_{\eta} \oplus \iota_{\eta}^{2} \jmath_{\eta} \oplus \omega_{\eta}^{2} \wp \jmath_{\eta} \oplus \varepsilon_{\eta} \ell_{\eta} \oplus \sigma_{\wp} \ell_{\eta}, v_{*}\right) \\
& \leq \tau_{\eta}^{2} \partial\left(v_{\eta}, v_{*}\right)+\kappa_{\eta}^{2} \partial\left(\wp v_{\eta}, v_{*}\right)+\iota_{\eta}^{2} \partial\left(\jmath_{\eta}, v_{*}\right)+\omega_{\eta}^{2} \partial\left(\wp \jmath_{\eta}, v_{*}\right) \\
& +\varepsilon_{\eta} \partial\left(\ell_{\eta}, v_{*}\right)+\sigma_{\eta} \partial\left(\wp \ell_{\eta}, v_{*}\right) \\
& \leq \tau_{\eta}^{2} \partial\left(v_{\eta}, v_{*}\right)+\kappa_{\eta}^{2}\left(\mu \partial\left(\wp v_{*}, v_{*}\right)+\partial\left(v_{\eta}, v_{*}\right)\right)+\iota_{\eta}^{2} \partial\left(\jmath_{\eta}, v_{*}\right)+\omega_{\eta}^{2}\left(\mu \partial\left(\wp v_{*}, v_{*}\right)\right. \\
& \left.+\partial\left(\jmath_{\eta}, v_{*}\right)\right)+\varepsilon_{\eta} \partial\left(\ell_{\eta}, v_{*}\right)+\sigma_{\eta}\left(\mu \partial\left(\wp v_{*}, v_{*}\right)+\partial\left(\ell_{n}, v_{*}\right)\right) \\
& \leq\left(\tau_{\eta}^{2}+\kappa_{\eta}^{2}+\iota_{\eta}^{2}+\omega_{\eta}^{2}\right) \partial\left(v_{\eta}, v_{*}\right)+\left(\varepsilon_{\eta}+\sigma_{\eta}\right) \partial\left(\ell_{\eta}, v_{*}\right) \\
& \leq\left(\tau_{\eta}^{2}+\kappa_{\eta}^{2}+\iota_{\eta}^{2}+\omega_{\eta}^{2}\right) \partial\left(v_{\eta}, v_{*}\right)+\left(\varepsilon_{\eta}+\sigma_{\eta}\right) \partial\left(\ell_{\eta}, v_{*}\right),
\end{aligned}
$$

by the above-mentioned standard three-step iteration algorithm,

$$
\begin{aligned}
\partial\left(v_{n+1}, v_{*}\right) & \leq\left(1-\left(\varepsilon_{\eta}+\sigma_{\eta}\right)\right) \partial\left(v_{\eta}, v_{*}\right)+\left(\varepsilon_{\eta}+\sigma_{\eta}\right) \partial\left(\ell_{\eta}, v_{*}\right) \\
& \leq \partial\left(v_{\eta}, v_{*}\right)-\left(\varepsilon_{\eta}+\sigma_{\eta}\right) \partial\left(v_{\eta}, v_{*}\right)+\left(\varepsilon_{\eta}+\sigma_{\eta}\right) \partial\left(\ell_{\eta}, v_{*}\right)
\end{aligned}
$$

This implies that,

$$
\begin{aligned}
\partial\left(v_{n+1}, v_{*}\right) & \leq \partial\left(v_{\eta}, v_{*}\right)-\left(\varepsilon_{\eta}+\sigma_{\eta}\right) \partial\left(v_{\eta}, v_{*}\right)+\left(\varepsilon_{\eta}+\sigma_{\eta}\right) \partial\left(\ell_{\eta}, v_{*}\right) . \\
& \leq \partial\left(v_{\eta}, v_{*}\right)+\left(\varepsilon_{\eta}+\sigma_{\eta}\right)\left(\partial\left(\ell_{\eta}, v_{*}\right)-\partial\left(v_{\eta}, v_{*}\right)\right) . \\
\partial\left(v_{n+1}, v_{*}\right)-\partial\left(v_{\eta}, v_{*}\right) & \leq\left(\varepsilon_{\eta}+\sigma_{\eta}\right)\left(\partial\left(\ell_{\eta}, v_{*}\right)-\partial\left(v_{\eta}, v_{*}\right)\right) . \\
\frac{\partial\left(v_{n+1}, v_{*}\right)-\partial\left(v_{\eta}, v_{*}\right)}{\left(\varepsilon_{\eta}+\sigma_{\eta}\right)} & \leq\left(\partial\left(\ell_{\eta}, v_{*}\right)-\partial\left(v_{\eta}, v_{*}\right)\right) .
\end{aligned}
$$

This implies that,

$$
\partial\left(v_{n+1}, v_{*}\right)-\partial\left(v_{\eta}, v_{*}\right) \leq \frac{\partial\left(v_{n+1}, v_{*}\right)-\partial\left(v_{\eta}, v_{*}\right)}{\left(\varepsilon_{\eta}+\sigma_{\eta}\right)} \leq\left(\partial\left(\ell_{\eta}, v_{*}\right)-\partial\left(v_{\eta}, v_{*}\right)\right)
$$

and hence, we have

$$
\partial\left(v_{n+1}, v_{*}\right) \leq \partial\left(\ell_{\eta}, v_{*}\right)
$$


Therefore,

$$
r \leq \lim _{n \rightarrow \infty} \partial\left(\ell_{\eta}, v_{*}\right)
$$

By using Equations (2.1) and (2.2), we have

$$
\begin{aligned}
r & =\lim _{n \rightarrow \infty} \partial\left(\ell_{\eta}, v_{*}\right) \\
& =\lim _{n \rightarrow \infty} \partial\left(\tau_{\eta}^{0} v_{\eta} \oplus \kappa_{\eta}^{0} \wp v_{\eta} \oplus \iota_{\eta}^{0} \jmath_{\eta} \oplus \omega_{\eta}^{0} \wp \jmath_{\eta}, v_{*}\right) \\
& =\lim _{n \rightarrow \infty}\left(\partial\left(\tau_{\eta}^{0} v_{\eta}+\kappa_{\eta}^{0} \wp v_{\eta}, v_{*}\right)+\left(\iota_{\eta}^{0}+\omega_{\eta}^{0}\right) \partial\left(\jmath_{\eta}, v_{*}\right)\right) \\
& =\lim _{n \rightarrow \infty}\left(\left(\tau_{\eta}^{0}+\iota_{\eta}^{0}+\omega_{\eta}^{0}\right) \partial\left(v_{\eta}, v_{*}\right)+\kappa_{\eta}^{0} \partial\left(\wp v_{\eta}, v_{*}\right)\right) \\
& =\lim _{n \rightarrow \infty}\left(\left(\tau_{\eta}^{0}+\iota_{\eta}^{0}+\omega_{\eta}^{0}\right) \partial\left(v_{\eta}, v_{*}\right)+\kappa_{\eta}^{0}\left(\mu \partial\left(\wp v_{*}, v_{*}\right)+\partial\left(v_{\eta}, v_{*}\right)\right)\right) \\
& =\lim _{n \rightarrow \infty}\left(\left(1-\kappa_{\eta}^{0}\right) \partial\left(v_{\eta}, v_{*}\right)+\kappa_{\eta}^{0} \partial\left(v_{\eta}, v_{*}\right)\right) . \\
& =\lim _{n \rightarrow \infty} \partial\left(v_{\eta}, v_{*}\right) .
\end{aligned}
$$

Using Equations (2.1) and (2.3) and the above-mentioned inequalities, we have

$$
\limsup _{n \rightarrow \infty} \partial\left(\ell_{\eta}, v_{*}\right) \leq \limsup _{n \rightarrow \infty} \partial\left(v_{\eta}, v_{*}\right)=r
$$

and hence, by Lemma 2, we have

$$
\lim _{n \rightarrow \infty} \partial\left(\wp v_{\eta}, v_{\eta}\right)=0
$$

Conversely, suppose that $\left\{v_{\eta}\right\}$ is bounded and $\lim _{n \rightarrow \infty} \partial\left(\wp v_{\eta}, v_{\eta}\right)=0$. Then, by Lemma $4\left\{v_{\eta}\right\}$ has a subsequence that is regular with respect to $\mathcal{B}_{s}$. Let $\left.v_{\{} \eta_{\kappa}\right\}$ be a subsequence of $\left\{v_{\eta}\right\}$ in such a way that $A\left(\mathcal{B}_{s},\left\{v_{\eta}\right\}\right)=v$. Hence, we have

$$
\begin{aligned}
\limsup _{n \rightarrow \infty} \partial\left(v_{\eta}, \wp v_{*}\right) & \leq \limsup _{n \rightarrow \infty}\left(\mu \partial\left(v_{\eta}, \wp v_{\eta}\right)+\partial\left(v_{\eta}, v\right)\right) \\
& \leq \limsup _{n \rightarrow \infty} \partial\left(v_{\eta}, v\right)
\end{aligned}
$$

As a consequence, the uniqueness of the asymptotic center ensures that $v$ is a fixed point of $\wp$ so this concludes the proof.

Now, we state and prove our main theorems in this section.

Theorem 2. Let $\mathcal{B}_{S}$ be a nonempty closed convex subset of a compete Busemann space $\mathcal{B}$ and $\wp: \mathcal{B}_{s} \rightarrow \mathcal{B}_{s}$ be a mapping satisfying condition $\left(\mathcal{E}_{\mu}\right)$. For an arbitrary chosen $v_{0} \in \mathcal{B}_{s}$, assume that $\left\{v_{\eta}\right\}$ is a sequence generated by a standard three-step iteration algorithm. Then $f_{\wp} \neq \varnothing$ and $\left\{v_{\eta}\right\} \Delta$-converges to a fixed point of $\wp$.

Proof. Since $f_{\wp} \neq \varnothing$, so by Lemma 8 , we have bounded $\left\{v_{\eta}\right\}$ and

$$
\lim _{n \rightarrow \infty} \partial\left(\wp v_{\eta}, v_{\eta}\right)=0
$$

Also, let

$$
\omega_{\omega}\left\{v_{\eta}\right\}:=\bigcup \mathcal{A}\left(v_{\eta_{\kappa}}\right)
$$

where the union is taken over all subsequences $\left\{v_{\eta_{\kappa}}\right\}$ of $\left\{v_{\eta}\right\}$. We claim that $\omega_{\omega}\left\{v_{\eta}\right\} \subset$ $f_{\wp}$. Considering $v_{*} \in \omega_{\omega}\left\{v_{\eta}\right\}$, then there is an existence of subsequence $\left\{v_{\eta_{\kappa}}\right\}$ of $\left\{v_{\eta}\right\}$ in such a way that $\mathcal{A}\left(\left\{v_{\eta}\right\}\right)=\left\{v_{*}\right\}$. Using Lemmas 3 and 5 there is an existence of subsequence $\left\{v_{\eta_{\kappa}}^{\prime}\right\}$ of $\left\{v_{\eta_{\kappa}}\right\}$ in such a way that $\Delta-\lim _{\eta \rightarrow \infty}\left\{v_{\eta_{\kappa}}^{\prime}\right\}=v_{*}^{\prime} \in \mathcal{B}_{s}$. Since $\lim _{\eta \rightarrow \infty} \partial\left(v_{\eta_{\kappa}}^{\prime}, \wp v_{\eta_{\kappa}}^{\prime}\right)=0$, then by Lemma $6 v^{\prime} \in f_{\wp}$. We claim that $v_{*}=v_{*}^{\prime}$. In contrast, 
since $\wp$ is a mapping satisfying condition $(\mathcal{E})$ and $v_{*} \in f_{\wp}$, then by Lemma 7 there is an existence of $\lim _{\eta \rightarrow \infty} \partial\left(v_{\eta}, v_{*}\right)$. Using the uniqueness of asymptotic centers, we have

$$
\begin{aligned}
\limsup _{n \rightarrow \infty} \partial\left(v_{\eta}, v_{*}^{\prime}\right) & <\limsup _{n \rightarrow \infty} \partial\left(v_{\eta_{\kappa}}^{\prime}, v_{*}\right) \\
& \leq \lim _{n \rightarrow \infty} \partial\left(v_{\eta_{\kappa}}, v_{*}\right) \\
& <\lim _{n \rightarrow \infty} \partial\left(v_{\eta_{\kappa}}^{\prime}, v_{*}^{\prime}\right) \\
& =\lim _{n \rightarrow \infty} \partial\left(v_{\eta}, v_{*}^{\prime}\right) \\
& =\lim _{n \rightarrow \infty} \partial\left(v_{\eta_{\kappa}}^{\prime}, v_{*}^{\prime}\right)
\end{aligned}
$$

which is a contradiction. So $v_{\eta_{\kappa}}^{\prime}=v_{\eta_{\kappa}} \in f_{\wp}$. To prove that $\left\{v_{\eta}\right\} \Delta$-converges to a fixed point of $\wp$, it is sufficient to show that $\omega_{\omega}\left\{v_{\eta}\right\}$ consists of exactly one point. Considering a subsequence $\left\{v_{\eta_{k}}\right\}$ of $\left\{v_{\eta}\right\}$. By Lemmas 3 and 5 there is existence of subsequence $\left\{v_{\eta_{\kappa}}^{\prime}\right\}$ of $\left\{v_{\eta_{\kappa}}\right\}$, which is how $\Delta-\lim _{\eta \rightarrow \infty}\left\{v_{\eta_{\kappa}}^{\prime}\right\}=v_{*}^{\prime} \in \mathcal{B}_{s}$. Let $\mathcal{A}\left(\left\{v_{\eta_{\kappa}}\right\}\right)=\left\{v_{*}\right\}$ and $\mathcal{A}\left(\left\{v_{\eta}\right\}\right)=v_{* *}$. We can conclude the explanation by proving that $v_{* *}=v_{*}$. On the contrary, since $\partial\left(v_{\eta}, v_{\eta_{\kappa}}^{\prime}\right)$ is convergent, then by the uniqueness of the asymptotic centers, we have

$$
\begin{aligned}
\limsup _{n \rightarrow \infty} \partial\left(v_{\eta}, v_{*}^{\prime}\right) & <\limsup _{n \rightarrow \infty} \partial\left(v_{\eta_{\kappa^{\prime}}}^{\prime} v_{* *}\right) \\
& \leq \lim _{n \rightarrow \infty} \partial\left(v_{\eta}, v_{* *}\right) \\
& <\lim _{n \rightarrow \infty} \partial\left(v_{\eta}, v_{*}^{\prime}\right) \\
& =\lim _{n \rightarrow \infty} \partial\left(v_{\eta_{\kappa^{\prime}}}^{\prime} v_{*}^{\prime}\right)
\end{aligned}
$$

which is a contradiction. Hence, $f_{\wp} \neq \varnothing$ and $\left\{v_{\eta}\right\} \Delta$-converges to a fixed point of $\wp$.

Theorem 3. Let $\mathcal{B}_{s}$ be a nonempty closed convex and complete Busemann space $\mathcal{B}$, and $\wp: \mathcal{B}_{s} \rightarrow$ $\mathcal{B}_{s}$ be a mapping verification condition $\left(\mathcal{E}_{\mu}\right)$. For an arbitrary chosen $v_{0} \in \mathcal{B}_{s}$, assume that $\left\{v_{\eta}\right\}$ is a sequence generated by a standard three-step iteration algorithm. Then $\left\{v_{\eta}\right\}$ converges strongly to a fixed point of $\wp$.

Proof. By Lemmas 7, 8 and Theorem 2, we have $f_{\wp} \neq \varnothing$ so by Lemma $8\left\{v_{\eta}\right\}$ is bounded and $\Delta$-converges to $v \in f_{\wp}$. Suppose on the contrary that $\left\{v_{\eta}\right\}$ does not converge strongly to $v$. By the compactness assumption, passing to subsequences if necessary, we may assume that there exists $v^{\prime} \in \mathcal{B}_{s}$ with $v^{\prime} \neq v$ such that $\left\{v_{\eta}\right\}$ converges strongly to $v^{\prime}$. Therefore,

$$
\lim _{n \rightarrow \infty} \partial\left(\wp v_{\eta}, v^{\prime}\right)=0 \leq \lim _{n \rightarrow \infty} \partial\left(\wp v_{\eta}, v\right) .
$$

Since $v$ is the unique asymptotic center of $\left\{v_{\eta}\right\}$, it follows that $v^{\prime}=v$, which is a contradiction. Hence, $\left\{v_{\eta}\right\}$ converges strongly to a fixed point of $\wp$.

\section{Conclusions}

The extension of the linear version of fixed point results to nonlinear domains has its own significance. To achieve the objective of replacing a linear domain with a nonlinear one, Takahashi [40] introduced the notion of a convex metric space and studied fixed point results of nonexpansive mappings in this direction. Since the standard three-step iteration scheme unifies various existing iteration schemes for different values of $\varepsilon_{\eta}, \sigma_{\eta}, \tau_{\eta}^{i}, \kappa_{\eta}^{i}, \omega_{\eta}^{i}$, and $\iota_{\eta}^{i}$ for $i=0,1,2$, existing results of the standard three-step iteration scheme including strong and $\Delta-$ convergence results in the setting of Busemann spaces satisfying condition $\mathcal{E}$ are generalized. 
Author Contributions: All authors have equally contributed to this work. All authors have read and agreed to the published version of the manuscript.

Funding: This work received no external funding.

Acknowledgments: The authors are very grateful to the anonymous reviewers for their constructive comments leading to the substantial improvement of the paper.

Conflicts of Interest: The authors declare no conflict of interest.

\section{References}

1. Babu, G.V.R.; Prasad, K. Mann iteration converges faster than Ishikawa iteration for the class of zamfirescu operators. Fixed Point Theory Appl. 2006, 2006, 49615. [CrossRef]

2. Busemann, H. Spaces with nonpositive curvature. Acta Math. 1948, 80, 259-310. [CrossRef]

3. Sosov, E.N. On analogues of weak convergence in a special metric space. Izvestiya Vysshikh Uchebnykh Zavedenii. Matematika 2004, $5,84-89$.

4. Lawaong, W.; Panyanak, B. Approximating fixed points of nonexpansive nonself-mappings in CAT(0) spaces. Fixed Point Theory Appl. 2010, 2010, 367274. [CrossRef]

5. Dhompongsa, S.; Inthakon, W.; Kaewkhao, A. Edelstien's method and Fixed point theorems for some generalized nonexpansive mappings. J. Math. Anal. Appl. 2009, 350, 12-17. [CrossRef]

6. Garcia-Falset, J.; Llorens-Fuster, E.; Suzuki, T. Fixed point theory for a class of generalized nonexpansive mappings. J. Math. Anal. Appl. 2011, 375, 185-195. [CrossRef]

7. Đukić, D.; Paunović, L.; Radenović, S. (). Convergence of iterates with errors of uniformly quasi-Lipschitzian mappings in cone metric spaces. Kragujev. J. Math. 2011, 35, 399-410.

8. Todorčević, V. Harmonic Quasiconformal Mappings and Hyperbolic Type Metrics; Springer Nature: Cham, Switzerland, 2019.

9. Xu, H.-K.; Altwaijry, N.; Chebbi, S. Strong convergence of mann's iteration process in Banach Spaces. Mathematics 2020, 8, 954. [CrossRef]

10. Ivanov, S.I. General local convergence theorems about the Picard iteration in arbitrary normed fields with applications to Super-Halley method for multiple polynomial zeros. Mathematics 2020, 8, 1599. [CrossRef]

11. Berinde, V. Iterative Approximation of Fixed Points. In Lecture Notes in Mathematics; Springer: Berlin/Heidelberg, Germany, 2007.

12. Ansari, Q.H.; Islam, M.; Yao, J.C. Nonsmooth variational inequalities on Hadamard manifolds. Appl. Anal. 2020, 99, 340-358. [CrossRef]

13. Hammad, H.A.; ur Rehman, H.; De la Sen, M. Advanced Algorithms and common solutions to variational inequalities. Symmetry 2020, 12, 1198. [CrossRef]

14. Dang, Y.; Sun, J.; Xu, H. Inertial accelerated algorithms for solving a split feasibility problem. J. Ind. Manag. Optim. 2017, 13, 1383-1394. [CrossRef]

15. Hammad, H.A.; ur Rehman, H.; De la Sen, M. Shrinking projection methods for accelerating relaxed inertial Tseng-type algorithm with applications. Math. Probl. Eng. 2020, 2020, 7487383. [CrossRef]

16. Tuyen, T.M.; Hammad, H.A. Effect of Shrinking Projection and CQ-Methods on Two Inertial Forward-Backward Algorithms for Solving Variational Inclusion Problems. In Rendiconti del Circolo Matematico di Palermo Series 2; Available online: https: / / doi.org/10.1007/s12215-020-00581-8 (accessed on 27 February 2021).

17. Papadopoulos, A. Metric Spaces, Convexity and Nonpositive Curvature. In IRMA Lectures in Mathematics and Theoretical Physics; European Mathematical Society: Strasbourg, France, 2005.

18. Sharma, N.; Mishra, L.N.; Mishra, V.N.; Almusawa, H. End point approximation of standard three-step multivalued iteration algorithm for nonexpansive mappings. Appl. Math. Inf. Sci. 2021, 15, 73-81.

19. Khamsi, M.A.; Kirk, W.A. An Introduction to Metric Spaces and Fixed Point Theory; John Wiley: New York, NY, USA, 2011.

20. Bridson, M.; Haefliger, A. Metric Spaces of Non-Positive Curvature; Springer: Berlin, Germany, 1999.

21. Kirk, W.A.; Panyanak, B. A concept of convergence in geodesic spaces. Nonlinear Anal. 2008, 68, 3689-3696. [CrossRef]

22. Kirk, W.A. Geodesic geometry and fixed point theory. In Seminar of Mathematical Analysis; Colecc. Abierta: Malaga, Spain, 2003.

23. Foertsch, T.; Lytchak, A.; Schroeder, V. Non-positive curvature and the Ptolemy inequality. Int. Math. Res. Not. 2007, 2007, rnm100.

24. Kirk, W.A. Geodesic geometry and fixed point theory II. In International Conference on Fixed Point Theory and Applications; Yokohama Publ.: Yokohama, Japan, 2004; pp. 113-142.

25. Bagherboum, M. Approximating fixed points of mappings satisfying condition (E) in Busemann space. Numer. Algorithms 2016, 71, 25-39. [CrossRef]

26. Kohlenbach, U. Some Logical theorems with applications in functional analysis. Tran. Am. Math. Soc. 2005, 357, 89-128. [CrossRef]

27. Noor, M.A. New approximation schemes for general variational inequalities. J. Math. Anal. Appl. 2000, 251, 217-229. [CrossRef]

28. Phuengrattana, W.; Suantai, S. On the rate of convergence of Mann, Ishikawa, Noor and SP-iterations for continuous functions on an arbitrary interval. J. Comput. Appl. Math. 2011, 235, 3006-3014. [CrossRef] 
29. Gursoy, F.; Karakaya, V. A Picard-S hybrid type iteration method for solving a differential equation with retarded argument. arXiv 2014, arXiv:1403.25-46.

30. Chugh, R.; Kumar, V.; Kumar, S. Strong Convergence of a new three step iterative scheme in Banach spaces. Am. J. Comput. Math. 2012, 2, 345-357. [CrossRef]

31. Abbas, M.; Nazir, T. A new faster iteration process applied to constrained minimization and feasibility problems. Mat. Vesn. 2014, 66, 223-234.

32. Sainuan, P. Rate of convergence of P-iteration and S-iteration for continuous functions on closed intervals. Thai J. Math. 2015, 13, 449-457.

33. Daengsaen, J.; Khemphet, A. On the Rate of Convergence of P-Iteration, SP-Iteration, and D-Iteration Methods for Continuous Non-decreasing Functions on Closed Intervals. Abstr. Appl. Anal. 2018, 2018, 7345401. [CrossRef]

34. Mann, W.R. Mean value methods in iteration. Proc. Am. Math. Soc. 1953, 4, 506-510. [CrossRef]

35. Ishikawa, S. Fixed points by a new iteration method. Proc. Am. Math. Soc. 1974, 44, 147-150. [CrossRef]

36. Goebel, K.; Reich, S. Uniform Convexity, Hyperbolic Geometry, and Nonexpansive Mappings; Marcel Dekker: New York, NY, USA; Basel, Switzerland, 1984.

37. Dhompongsa, S.; Kirk, W.A.; Sims, B. Fixed points of uniformly lipschitzian mappings. Nonlinear Anal. 2006, 65, 762-772. [CrossRef]

38. Kirk, W.; Shahzad, N. Fixed Point Theory in Distance Spaces; Springer: Cham, Switzerland; Heidelberg, Germany; New York, NY, USA; Dordrecht, The Netherlands; London, UK, 2014.

39. Ullah, K.; Khan, B.A.; Ozer, O.; Nisar, Z. Some Convergence Results Using $K_{*}$ Iteration Process in Busemann Spaces. Malays. J. Math. Sci. 2019, 13, 231-249.

40. Takahashi, T. A convexity in metric spaces and nonexpansive mappings. Kodai Math. Semin. Rep. 1970, 22, 142-149. [CrossRef] 